\title{
GENERALIZED CONSERVATION LAWS
}

\author{
A. P. STONE ${ }^{1}$
}

1. Introduction. Let $\left(u^{1}, u^{0}, \cdots, u^{n}\right)$ form a (local) coordinate system belonging to the ring $A$ of germs of analytic functions at some point $P$ of an analytic manifold, and let $u^{0}(P)=u^{1}(P)=\cdots=u^{n}(P)$ $=0$. Denote by $\mathcal{E}$ the localization of the module of differential forms on the manifold; $\mathcal{E}$ is in fact a free $A$-module with generators $\left(d u^{0}, d u^{1}, \cdots, d u^{n}\right)$ where $d$ is the operation of exterior differentiation. If $h$ and $k$ are endomorphisms of $\varepsilon$, we shall call an element $\theta \in \mathcal{E}$ a conservation law for $h$ and $k$ if and only if $\theta, h \theta$, and $k \theta$ are all (locally) exact forms. This definition generalizes the idea of a conservation law for a single endomorphism $h$. The reader should see [4] or [5] for example.

The problem considered in this paper is that of determining all conservation laws $\theta \in \varepsilon$ for a given $h$ and $k$. As in [6] it is convenient to impose some conditions on $h$ and $k$. Specifically we will require that $h$ and $k$ have distinct eigenvalues and certain (Nijenhuis) tensors derived from $h$ and $k$ vanish. For example, the vanishing of the tensor $[h, h]$ is an integrability condition which, with appropriate hypotheses, ensures the existence of a basis of conservation laws for $\varepsilon$.

The preceding problem is identical to the following one concerning systems of linear homogeneous partial differential equations in three independent variables. Let the matrix $\left(h_{j}^{i}\right)$ represent $\boldsymbol{h}$ and the matrix $\left(k_{j}^{i}\right)$ represent $k$ with respect to the basis $\left(d u^{0}, d u^{1}, \cdots, d u^{n}\right)$ of $\varepsilon$. If one considers the system

$$
\frac{\partial u^{i}}{\partial t}+h_{\alpha}^{i} \frac{\partial u^{\alpha}}{\partial x}+k_{\alpha}^{i} \frac{\partial u^{\alpha}}{\partial y}=0, \quad i=0,1, \cdots, n,
$$

where the use of Greek indices indicates a summation from 0 to $n$, and $\left(h_{j}^{i}\right)$ and $\left(k_{j}^{i}\right)$ depend only on $u^{0}, u^{1}, \cdots, u^{n}$, the problem is one of finding a suitable nonsingular matrix which multiplies the matrix equation (1.1) on the left so as to obtain a new system

$$
\frac{\partial}{\partial t} U+\frac{\partial}{\partial x} V+\frac{\partial}{\partial y} W=0
$$

where $U, V$, and $W$ are column vectors depending on $u^{0}, u^{1}, \cdots, u^{n}$.

Presented to the Society, January 25, 1967 under the title Analytic conservation laws; received by the editors July 23, 1966.

1 This research was supported in part by NSF Grant GP6560. 
The system (1.2) is a system of conservation laws. We note that in particular the system (1.1) is said to contain a single conservation law

$$
\frac{\partial f}{\partial t}+\frac{\partial g}{\partial x}+\frac{\partial h}{\partial y}=0
$$

provided that functions $v_{0}, v_{1}, \cdots, v_{n}$ of $u^{0}, u^{1}, \cdots, u^{n}$ can be found such that

$$
v_{\alpha} \frac{\partial u^{\alpha}}{\partial t}=\frac{\partial f}{\partial t}, \quad v_{\alpha} h_{\beta}^{\alpha} \frac{\partial u^{\beta}}{\partial x}=\frac{\partial g}{\partial x}
$$

and

$$
v_{\alpha} k_{\beta}^{\alpha} \frac{\partial u^{\beta}}{\partial y}=\frac{\partial h}{\partial y} .
$$

Since many properties are known for systems of partial differential equations which contain conservation laws it is of interest to know when systems such as (1.1) contain conservation laws or can be expressed entirely in terms of conservation laws. For example, in the study of hyperbolic partial differential equations one finds that the notion of a weak solution of (1.2) depends on the fact that (1.2) is a system of conservation laws (see [2]).

2. Preliminaries. In this section some definitions which appear elsewhere are repeated. As in $\$ 1$, let $\varepsilon$ be the free $A$-module on $(n+1)$ generators $\left(d u^{0}, d u^{1}, \cdots, d u^{n}\right)$. One defines $d u^{i}$ by giving its value on an element $\partial / \partial u^{i}$ of $E=\operatorname{Hom}(\mathcal{E}, A)$. That is, $\left\langle\partial / \partial u^{i}, d u^{i}\right\rangle=\delta_{j}^{i} \in A$ for $i, j \in(0,1, \cdots, n)$. The elements of $E$ are called vector fields even though $A$ is a ring of germs. Since $E$ is also a free $A$-module on generators $\left(\partial / \partial u^{0}, \partial / \partial u^{1}, \cdots, \partial / \partial u^{n}\right)$, any element $M \in E$ can be written uniquely in the form $M=m_{\alpha} \partial / \partial u^{\alpha}$ for functions $m_{i} \in A$. Vector fields are derivations of $A$ into itself: that is, for any $a, b \in A$ and $M \in E, M(a b)=a(M b)+b(M a)$.

The elements of $E$ will be denoted by $L, M, \cdots$, while the elements of $\varepsilon$ will be denoted by $\theta, \phi, \ldots$, and so forth. An endomorphism $h$ of $\varepsilon$ will be distinguished from its adjoint by writing $L h$ when $h$ acts on elements of $E$ and $h \theta$ when $h$ acts on elements of $\varepsilon$. That is, $\langle L h, \theta\rangle=\langle L, h \theta\rangle$ for any $L \in E$ and $\theta \in \mathcal{E}$.

If $\Lambda^{*} \mathcal{E}$ denotes the exterior algebra generated by $\mathcal{E}$, we note that an element $h \in \operatorname{Hom}_{A}(\mathcal{E}, \mathcal{E})$ induces homomorphisms of $\Lambda^{*} \mathcal{E}$ which is a direct sum

$$
\Lambda^{*} \mathcal{E}=\Lambda^{0} \mathcal{E} \oplus \Lambda^{1} \mathcal{E} \oplus \cdots \oplus \Lambda^{n} \mathcal{E}
$$


where $\Lambda^{0} \mathcal{E}=A$ and $\Lambda^{1} \mathcal{E}=\mathcal{E}$. The induced transformations which are of primary interest to us are defined by

$$
\begin{aligned}
& h^{(1)}(\theta \wedge \phi)=h \theta \wedge h \phi, \\
& h^{(2)}(\theta \wedge \phi)=h \theta \wedge \phi+\theta \wedge h \phi,
\end{aligned}
$$

for any $\theta, \phi \in \mathcal{E}$. The map $h^{(0)}$ is the identity on $\mathcal{E} \wedge \mathcal{E}$. The equation (2.1) defines a homomorphism of $\Lambda^{*} E$, and (2.2) defines a derivation. That these maps are well defined may easily be verified.

The Nijenhuis torsion $[h, h]$ of $h$ is defined for any $\theta \in \varepsilon$ as an element of $\operatorname{Hom}_{A}(\mathcal{E}, \mathcal{E} \wedge \mathcal{E})$ by the formula

$$
[h, h] \theta=-h^{(2)} d \theta+h^{(1)} d h \theta-h^{(0)} d h^{2} \theta .
$$

Again it is easy to establish that $[h, h]$ is well defined. If $[L, M]$ denotes the Lie bracket of any two vector fields $L$ and $M$, then a dual characterization of $[h, h]$ as an element of $\operatorname{Hom}_{A}(E \wedge E, E)$ may also be obtained by setting

$$
\begin{aligned}
& (L \wedge M)[h, h] \\
& \quad=[L, M] h^{2}+[L h, M h]-[L h, M] h-[L, M h] h .
\end{aligned}
$$

It should be observed that if $h$ is singular one can always obtain a nonsingular transformation $h^{*}$ such that $[h, h]=\left[h^{*}, h^{*}\right]$ simply by setting $h^{*}=h+\alpha I$, where $\alpha$ is a suitable nonzero constant.

Finally, for any endomorphisms $h$ and $k$ of $\varepsilon$ the differential concomitant $[h, k] \in \operatorname{Hom}(\varepsilon, \varepsilon \wedge \varepsilon)$ for any $\theta \in \mathcal{E}$ is defined by the formula

$$
\begin{aligned}
{[\boldsymbol{h}, \boldsymbol{k}] \theta=\frac{1}{2}\left\{-\left[h^{(1)} k^{(1)}-(h k)^{(1)}\right] d \theta\right.} \\
\left.+\left[h^{(1)} d \boldsymbol{k} \theta+k^{(1)} d \boldsymbol{h} \theta\right]-[d \boldsymbol{h} \boldsymbol{k} \theta+d \boldsymbol{k} \boldsymbol{h} \theta]\right\} .
\end{aligned}
$$

A dual formula which appears in [1] can also be established. It is given for any $L$ and $M \in E$ by

$$
\begin{aligned}
&(L \wedge M)[h, k]= \frac{1}{2}\{[L, M] h k+[L, M] k h+[L h, M k] \\
&+[L k, M h]-[L h, M] k-[L k, M] h \\
& \\
&-[L, M h] k-[L, M k] h\} .
\end{aligned}
$$

3. The conservation law problem. The result obtained in this section can be'stated as follows. Let $h$ and $k \in \operatorname{Hom}_{A}(\varepsilon, \varepsilon)$ and the eigenvalues of $h$ and $k$ respectively be distinct. If $[h, h]$ and $[h, k]$ vanish and $h k=k h$, then $\theta$ is a conservation law for $h$ if and only if $\theta$ is a conservation law for $\boldsymbol{k}$. Since the problem of obtaining conservation laws for a single endomorphism has been treated in several papers (see 
$[4],[5]$ and [6]), one is then in a position to apply the results of these investigations and obtain conservation laws for a collection of endomorphisms $\boldsymbol{h}_{\mathbf{1}}, \boldsymbol{h}_{2}, \cdots, \boldsymbol{h}_{\boldsymbol{p}}$ under appropriate hypotheses.

If the eigenvalues $\lambda_{0}, \lambda_{1}, \cdots, \lambda_{n}$ of $h$ are distinct, then $h$ is cyclic on $\mathcal{E}$ (its adjoint is also cyclic on $E$ ) and the assumption that $\boldsymbol{h} \boldsymbol{k}=\boldsymbol{k} \boldsymbol{h}$ implies $\boldsymbol{k}$ is a polynomial in $\boldsymbol{h}$. That is, $\boldsymbol{k}$ has the form

$$
\boldsymbol{k}=f(\boldsymbol{h})=b_{0} I+b_{1} \boldsymbol{h}+\cdots+b_{n} \boldsymbol{h}^{n}, \quad b_{i} \in A,
$$

and any eigenvectors of $\boldsymbol{h}$ are also eigenvectors of $\boldsymbol{k}$ while

$$
\beta_{i}=f(\lambda i)=b_{0}+b_{1} \lambda_{i}+\cdots+b_{n} \lambda_{i}^{n}, \quad i \in(0,1, \cdots, n),
$$

are the eigenvalues of $\boldsymbol{k}$.

THeOREM 3.1. If the eigenvalues $\lambda_{0}, \cdots, \lambda_{n}$ of $h$ are distinct, if $h k=k h$ and if $[h, h]=[h, k]=0$, then there exist coordinates $v^{0}, \ldots$, $v^{n}$ such that $\partial / \partial v^{i}(i=0,1, \cdots, n)$ are eigenvector fields of $h$ and $k$, and the corresponding eigenvalues $\lambda_{i}$ and $\beta_{i}$ of $h$ and $k$ respectively are functions of $v^{i}$ alone.

Proof. Let $L_{i}$ and $L_{j}(i \neq j)$ be eigenvector fields of $\boldsymbol{h}$. Since $h k=k h, L_{i}$ and $L_{j}$ are also eigenvector fields of $k$. Thus if one sets $L_{i} h=\lambda_{i} L_{i}, L_{j} h=\lambda_{j} L_{j}, L_{i} k=\beta_{i} L_{i}$, and $L_{j} k=\beta_{j} L_{j}$ and applies equation (2.6) the result is

$$
\begin{aligned}
2\left(L_{i} \wedge L_{j}\right)[h, k]= & {\left[L_{i}, L_{j}\right]\left\{\left(h-\lambda_{i} I\right)\left(k-\beta_{i} I\right)+\left(h-\lambda_{j} I\right)\left(k-\beta_{i} I\right)\right\} } \\
& +\left\{\left(\lambda_{i}-\lambda_{j}\right)\left(L_{j} \beta_{i}\right)+\left(\beta_{i}-\beta_{j}\right)\left(L_{j} \lambda_{i}\right)\right\} L_{i} \\
& +\left\{\left(\lambda_{i}-\lambda_{j}\right)\left(L_{i} \beta_{j}\right)+\left(\beta_{i}-\beta_{j}\right)\left(L_{i} \lambda_{j}\right)\right\} L_{j} .
\end{aligned}
$$

In the event that $\boldsymbol{h}=\boldsymbol{k}$ the above relation reduces to

$$
\begin{aligned}
\left(L_{i} \wedge L_{j}\right)[\boldsymbol{h}, \boldsymbol{h}]=\left[L_{i}, L_{j}\right]\left\{\left(\boldsymbol{h}-\lambda_{i} I\right)\right. & \left.\left(\boldsymbol{h}-\lambda_{j} I\right)\right\} \\
& +\left(\lambda_{i}-\lambda_{j}\right)\left\{\left(L_{j} \lambda_{i}\right) L_{i}+\left(L_{i} \lambda_{j}\right) L_{j}\right\},
\end{aligned}
$$

and the existence of coordinates $v^{0}, \ldots, v^{n}$ such that $\partial / \partial v^{0}, \ldots$, $\partial / \partial v^{n}$ are eigenvector fields (cf. [1] and [3]) is a consequence of $[h, h]=0$. Moreover since $\lambda_{i} \neq \lambda_{j}$ it follows that $\left(L_{i} \lambda_{j}\right)=0$ for $i \neq j$ and consequently $\lambda_{i}$ depends on $v^{i}$ alone.

If one returns to the original formula for $2\left(L_{i} \wedge L_{j}\right)[h, k]$ it is now clear that the vanishing of $[h, h]$ yields

$$
2\left(L_{i} \wedge L_{j}\right)[h, k]=\left(\lambda_{i}-\lambda_{j}\right)\left\{\left(L_{j} \beta_{i}\right) L_{i}+\left(L_{i} \beta_{j}\right) L_{j}\right\},
$$

and hence $\beta_{i}$ does not depend on $v^{j}$ if $[h, k]$ vanishes.

Theorem 3.2. Let $\boldsymbol{h}$ have distinct eigenvalues $\boldsymbol{\lambda}_{j}$. If $\boldsymbol{h} \boldsymbol{k}=\boldsymbol{k} \boldsymbol{h}$ and 
$[\boldsymbol{h}, \boldsymbol{h}]=[\boldsymbol{h}, \boldsymbol{k}]=0$ and $\theta \in \mathcal{E}$ is a conservation law for $\boldsymbol{h}$, then it is also a conservation law for $\boldsymbol{k}$.

Proof. The conditions of the theorem guarantee the existence of a basis of eigenforms $\left(d v^{0}, d v^{1}, \cdots, d v^{n}\right)$ of $\varepsilon$, and any conservation law for $\boldsymbol{h}$ must have the form $\theta=\alpha_{0} d v^{0}+\cdots+\alpha_{n} d v^{n}$, where each function $\alpha_{j}$ depends on $v^{j}$ alone. Consequently

$$
\boldsymbol{k} \theta=\alpha_{0} \beta_{0} d v^{0}+\alpha_{1} \beta_{1} d v^{1}+\cdots+\alpha_{n} \beta_{n} d v^{n}
$$

and hence by Theorem 3.1 it follows that $d(k \theta)=0$. Since closed forms are also (locally) exact, $\theta$ is then a conservation law for $k$.

It should be remarked that the vanishing of $[k, k]$ is a consequence of the hypotheses imposed on $h$ and $k$ in Theorems 3.1 and 3.2. This fact should be clear from the following argument. Since one can always obtain a generator $\theta$ of $\varepsilon$ which is also a conservation law (for $h$ ) simply by setting $\theta=d v^{0}+d v^{1}+\cdots+d v^{n}$ and thereby obtain a basis $\left(\theta, h \theta, h^{2} \theta, \cdots, h^{n} \theta\right)$ of $\mathcal{E}$, it suffices to compute $[k, k]\left(h^{i} \theta\right)$ for $i=0,1, \cdots, n$. The vanishing of $[k, k]$ is then a consequence of the fact that $d\left(\boldsymbol{k}^{i} h^{i} \theta\right)=d\left(\beta_{\alpha}^{j} \lambda_{\alpha}^{i} d v^{\alpha}\right)=0$ for any pair $(i, j)$ of nonnegative integers.

If the additional condition that $k$ has distinct eigenvalues $\beta_{j}$ is imposed, then one can obtain the following theorem.

TheOREM 3.3. If $h$ and $k$ have distinct eigenvalues $\lambda_{j}$ and $\beta_{j}$ respectively, $h k=k h$, and $[h, h]=[h, k]=0$, then any conservation law for $k$ is also a conservation law for $h$.

Proof. Since $\left(d v^{0}, d v^{1}, \cdots, d v^{n}\right)$ is a basis of eigenforms for $\mathcal{E}$, any conservation law for $\boldsymbol{k}$ will have the form

$$
\phi=\gamma_{0} d v^{0}+\gamma_{1} d v^{1}+\cdots+\gamma_{n} d v^{n},
$$

where the functions $\gamma_{i}$ may be functions of more than one variable $v^{i}$. It suffices to show that $\gamma_{i}=\gamma_{i}\left(v^{i}\right)$, for then clearly $d(h \phi)=0$. To simplify the notation, let us set $\partial \gamma_{\jmath} / \partial v^{i}=\gamma_{j, i}$ and use Greek indices $\alpha$ and $\sigma$ to indicate summations. Then the hypotheses that $d \phi$ $=d(k \phi)=0$ lead to the conditions

(i) $d \phi=\left(\gamma_{\sigma, \alpha}-\gamma_{\alpha, \sigma}\right) d v^{\alpha} \wedge d v^{\sigma}=0$ and

(ii) $d(k \phi)=\left(\beta_{\sigma} \gamma_{\sigma, \alpha}-\beta_{\alpha} \gamma_{\alpha, \sigma}\right) d v^{\alpha} \wedge d v^{\sigma}=0$

which may be rewritten as

(i') $\gamma_{i, j}=\gamma_{j, i}$ and

(ii') $\beta_{i} \gamma_{i, j}=\beta_{j} \gamma_{j, i}$

where $i \neq j$. Since $\beta_{j}$ are distinct, it follows that $\gamma_{i, j}=0$ or $\gamma_{i}=\gamma_{i}\left(v^{i}\right)$. 
4. Conclusion. Since it is known that conservation laws exist (locally) for a single endomorphism $h$ in the case that $h$ has distinct eigenvalues and $[h, h]=0$, one then observes that this class of conservation laws agrees with the class of conservation laws for a given $\boldsymbol{k}$ if the conditions $[\boldsymbol{h}, \boldsymbol{k}]=0, \boldsymbol{k}=\boldsymbol{h} \boldsymbol{k}$, and $\boldsymbol{k}$ has distinct eigenvalues are additionally imposed. That this class can in fact be quite large may be seen from equation (2.3), which says that if $\theta$ is a conservation law for $h$, then $h^{i} \theta$ is also a conservation law for any positive integer $i$.

The author is indebted to the referee for his helpful comments.

\section{REFERENCES}

1. A. Frölicher and A. Nijenhuis, Theory of vector-valued differential forms. I, Nederl. Akad. Wetensch. Proc. 59 (1956), 338-359.

2. P. Lax, Hyperbolic systems of conversion laws. II, Comm. Pure Appl. Math. 10 (1957), 537-566.

3. A. Nijenhuis, $X_{n-1}$-forming sets of eigenvectors, Nederl. Akad. Wetensch. Proc. 54 (1951), 200-212.

4. H. Osborn, The existence of conservation laws. I, Ann. of Math. 69 (1959), 105-118.

5. - Les lois de conservation, Ann. Inst. Fourier (Grenoble) 14 (1964), 71-82.

6. A. Stone, The existence of conservation laws on analytic manifolds, $\mathrm{Ph}$. D. thesis, Univ. of Illinois, Chicago, 1965.

UNIVERSITY OF ILLINOIS 